\author{
Jing-fu Liu ${ }^{1}$ \\ Xia Liang ${ }^{1,2}$ \\ Gui-bin Jiang ${ }^{1}$ \\ Ya-qi Cai ${ }^{1}$ \\ Qing-xiang Zhou', 2 \\ Guo-guang Liu²
}

${ }^{1}$ Key Laboratory of Environmental Chemistry and Ecotoxicology,

Research Center for Eco-

Environmental Sciences,

Chinese Academy of Sciences,

P. O. Box 2871, Beijing 100085,

China

${ }^{2}$ Key Laboratory of Environmental

Science and Engineering,

College of Environmental

Sciences and Chemistry, Henan

Normal University, Xinxiang

453002, Henan, China

\section{High performance liquid chromatography determination of 4-nonylphenol, 4-tert-octylphenol, and their short ethoxyl chain polyethoxylates in water samples using a microporous membrane liquid-liquid extraction sample pretreatment technique}

Microporous membrane liquid-liquid extraction (MMLLE) sample pretreatment, coupled with reversed-phase high performance liquid chromatography with fluorescence detection (HPLC-FLD) was proposed for the determination of alkylphenols (APs), including 4-nonylphenol (4-NP) and 4-tert-octylphenol (4-t-OP), and their short ethoxyl chain polyethoxylates (SEC-APEOs) in environmental aqueous samples. After MMLLE, 4-NP, 4-t-OP, and SEC-APEOs were trapped in hexane used as acceptor. The hexane with enriched analytes was purged to dryness with nitrogen gas. Methanol $(0.6 \mathrm{~mL})$ and then water $(1.4 \mathrm{~mL})$ were added to dissolve the residue and the analytes were subsequently focused onto a $\mathrm{C}_{18}$ precolumn prior to injection onto the analytical column for separation and detection by reversed-phase HPLC-FLD. The proposed procedure permits convenient determination of APs and SEC-APEOs, which have similar endocrine activity and appear as a single peak in reversed-phase HPLC. The detection limits for 4-NP and 4-t-OP were 0.1 and $0.05 \mu \mathrm{g} / \mathrm{L}$, respectively. Four samples including tap water, river water, and effluent from a sewage treatment works water were analyzed by the proposed method and the recoveries at $0.5 \mu \mathrm{g} / \mathrm{L}$ spiked level were in the range of $74-110 \%$.

Key Words: Microporous membrane liquid-liquid extraction; HPLC; 4-nonylphenol; 4-tert-octylphenol; Aqueous samples

Received: September 17, 2002; revised: January 6, 2003; accepted: January 22, 2003

DOI 10.1002/jssc.200301455

\section{Introduction}

4-Nonylphenol (4-NP), 4-tert-octylphenol (4-t-OP), and their short ethoxyl chain polyethoxylates (SEC-APEOs) occurring in the aquatic environment are mainly degradation products of 4-nonylphenol polyethoxylates (NPEOs) and 4-tert-octylphenol polyethoxylates (OPEOs) [1], which are a group of non-ionic surfactants widely used as detergents, wetting agents, emulsifiers, and stablizers [2]. 4-NP, 4-t-OP, and SEC-APEOs (chemical structures shown in Figure 1), are more persistent than their parent compounds and have been found to elicit estrogenic activities [3, 4]. Moreover, these compounds may persist in aquatic sediments where they may remobilize and bioaccumulate in the food chain [5]. Therefore, the development of analytical methods for these contaminants has recently attracted considerable attention and various

Correspondence: Gui-bin Jiang, Research Center for Eco-Environmental Sciences, Chinese Academy of Sciences, P. O. Box 2871, Beijing 100085, China. Phone: +86 1062849334.

Fax: +86 10 62923563. E-mail: gbjiang @ mail.rcees.ac.cn.

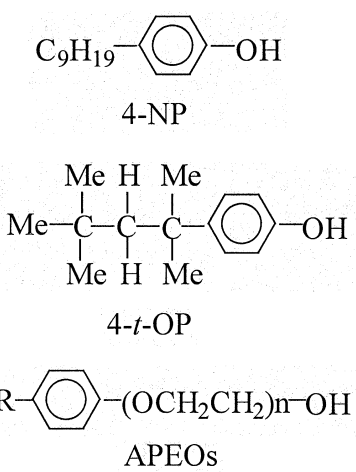

Figure 1. Chemical structures of 4-NP, 4-t-OP, and APEOs.

aspects of their analytical chemistry have been reviewed $[6,7]$.

The relatively low concentration levels and the complex matrices in which these analytes occur in environmental samples usually necessitate sample enrichment and clean up procedures, and various sample pretreatment procedures have been reported. Although solid phase 
extraction (SPE) has become the most widely reported technique for pretreatment of water samples containing these contaminants [8-12], liquid-liquid extraction (LLE) $[7,13]$ is still used for this purpose in routine analysis.

Recently, a number of different membrane extraction techniques have been suggested as alternatives to SPE and LLE [14, 15]. However, no study on the application of membrane techniques to the enrichment of alkylphenols (APs) and alkylphenol polyethoxylates (APEOs) has been reported.

Because gas chromatography (GC) usually needs a derivatization step and is not applicable to the analysis of the highly ethoxylated APEOs, HPLC is the most commonly used technique for the determination of these APs and APEOs. So far, HPLC with ultraviolet $[16,17]$, electrochemical [18], fluorescence [8-9], and MS [11, 12] detection has been used for the determination of these compounds. HPLC is able to separate and quantitate the various homologues and oligomers according to the length of their alkyl and ethoxylate chains. Normal-phase HPLC resolves the ethoxylate oligomers, whereas reversedphase HPLC provides information about the alkyl chain length [8]. APs and SEC-APEOs have similar endocrine activities, and can be determined as a total in environmental contamination evaluation. Therefore, it is useful to develop sample pretreatment procedures that can separate APs and SEC-APEOs from long ethoxyl chain APEOs (LEC-APEOs).

The objective of this work was to study the suitability of microporous membrane liquid-liquid extraction (MMLLE) [15] for enrichment of 4-NP, 4-t-OP, and their SECAPEOs in aquatic environmental samples, and the coupling of MMLLE with reversed-phase HPLC-FLD for the determination of the above target compounds. MMLLE is an aqueous-organic extraction system with a hydrophobic PTFE microporous membrane to block off the organic acceptor and the aqueous samples. The organic solvent fills the pores of the membrane and thus contacts the aqueous sample and extracts the target analyte.

\section{Experimental}

\subsection{Reagents and samples}

All reagents were of analytical-reagent grade and deionized water was used throughout. 4-NP and 4-t-OP were obtained from Tokyo Kasei Kogyo Co. LTD, Japan. NP6.5EO, NP10EO, and NP15EO are nonylphenol polyethoxylates with average ethoxy units of $6.5,10$, and 15 , respectively. NP6.5EO and NP10EO are industrial products purchased from Henkel (Gemany) and Rhodia (France), respectively. NP15EO was purchased from Tokyo Kasei, Japan. Octylphenol polyethoxylate (OP10EO, commercial name Triton X 100, pure) was pur- chased from Carl Roth KG Chemiche Fabrik Karlsruhe. Standard stock solutions $\left(1000 \mu \mathrm{g} \mathrm{mL}^{-1}\right)$ of these compounds were prepared in methanol. Working solutions were prepared daily by appropriate dilution of the stock solutions with de-ionized water. HPLC-grade methanol and acetonitrile, and Multisolvent-grade $n$-hexane (96\%) were purchased from Scharlace Chemie SA, Barcelona, Spain. Sodium hydroxide and sodium chloride were analytical grade reagents (Beijing Chemicals Corporation, Beijing, China).

A tap water sample was collected from the water tap in our laboratory, river water samples were taken from the Beijing section of the Yongding River valley, and the wastewater sample was effluent collected at the Gaobeidian sewage treatment works in the eastern part of Beijing. The samples were filtered through a Millipore cellulose membrane of $0.45 \mu \mathrm{m}$ pore size and stored in glass containers at $4^{\circ} \mathrm{C}$.

\subsection{Instrumentation}

The MMLLE equipment shown in Figure 2 consisted of a MMLLE unit, two six-port injection valves, a piston pump (MiniPump, Laboratory Data Control, Division of Milton Roy Company) and a glass syringe. The custom-made MMLLE unit consisted of a PTFE block (PB), a PTFE membrane $(P M)$, and an aluminum support plate $(A B)$. The PTFE block was machined from two blocks of Teflon by cutting two Archimedes' spiral grooves (SC) on the opposite faces of the two blocks. The grooves have identical dimensions of $0.3 \mathrm{~mm}$ depth, $2.0 \mathrm{~mm}$ width, and $160 \mathrm{~cm}$ length. The membrane (Fluoropore FG PTFE membrane with average pore size $0.2 \mu \mathrm{m}$, porosity 0.7 ; Millipore Co., Bedford, MA, USA) was clamped tightly and evenly between the planar surfaces of the blocks by 8 screws. Thus, two channels (the acceptor channel and the donor channel) with the same volume of $960 \mu \mathrm{L}$ were obtained. To make the device more rigid, each PTFE block was backed by an aluminum block. To prevent any
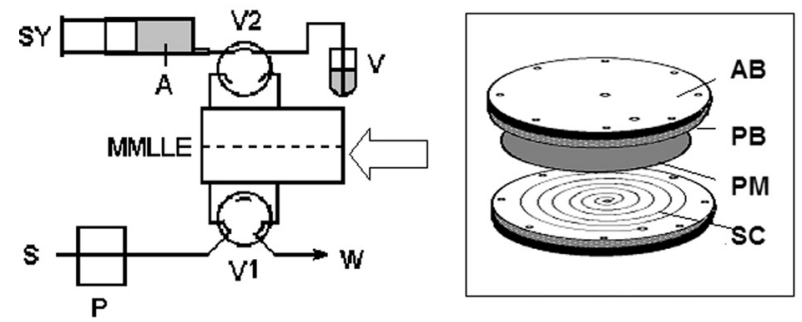

Figure 2. Schematic diagram of the MMLLE setup. P, pump; V1, V2, valves; MMLLE, microporous membrane liquid-liquid extraction device; SY, glass syringe; V, vial; S, sample; A, accepter (hexane); W, waste; $A B$, aluminum backer; $P B$, PTFE block; PM, PTFE membrane; SC, spiral channel. See text for details. 
of its ingredients from being dissolved in hexane (acceptor) and thus interfering with the determination, the polyethylene backing of the PTFE membrane was manually torn away from the circular edge of the membrane. One six-port injection valve was used to control the entry of sample solution into the donor of the extraction system, while another one was used to load and take out the organic solvent and keep the channel airtight during the extraction procedure.

The HPLC equipment used included an Agilent 1100 Series IsoPump and an Agilent 1100 Series FLD detector. The sampling loop of a 7725 injector (Rheodyne, USA) was replaced by a $\mathrm{C}_{18}$ precolumn $(16 \times 4.0 \mathrm{~mm}$ ID, Three Dimension Chromatography Co., Tianjin, China), and the original out-port (port 6) of the 7725 injector was connected to a piston pump (MiniPump, Laboratory Data Control, Division of Milton Roy Company) to introduce sample solutions into the precolumn. The original female injection port was extended to the waste with PTFE tubing. An Agilent Zorbax Eclipse $\mathrm{XDB}^{-\mathrm{C}_{8}}$ column $(150 \times$ $4.6 \mathrm{~mm}$, particle size $5 \mu \mathrm{m}$ ) was used as analytical column. For all compounds of interest, the fluorescence detector was set as follows: $220 \mathrm{~nm}$ excitation, $315 \mathrm{~nm}$ emission. A personal computer equipped with an Agilent ChemStation program for LC and LC/MS systems was used to process chromatographic data. The mobile phase was a mixture of acetonitrile and water $(70+30$, $v+v)$.

\subsection{Procedure}

The overall MMLLE procedure is best described by reference to Figure 2. Sample solution (S) was introduced into donor channel of the system by piston $(P)$ by switching $V 1$ to the sampling position. V2 was switched to the bypass position when the sample was introduced into the MMLLE device, so the hexane acceptor, delivered by a manually controlled syringe, remained stagnant in the acceptor channel and the analytes were trapped in it.

After a certain time, V1 was switched to the bypass position but V2 was switched to the injection position, and the syringe was manually pushed to transport hexane acceptor $(A)$, with the analytes that have been enriched in the acceptor channel, to a vial. The acceptor in the vial was purged to dryness with high purity nitrogen. Then, the analytes were dissolved in methanol $(0.6 \mathrm{~mL})$, and water $(1.4 \mathrm{~mL})$ was added and mixed. Subsequently, the piston pump connected to the out-port of the 7725 injector was actuated to transport the mixture onto the $\mathrm{C}_{18}$ precolumn where the analytes were focused; $30 \%(\mathrm{v} / \mathrm{v})$ methanol $(2 \times 3 \mathrm{~mL})$ was added to the vial to ensure complete transfer of the analytes onto the precolumn. Finally, the analytes focused on the precolumn were injected onto the analytical column for separation and detection.

\section{Results and discussion}

\subsection{HPLC separation}

Different mobile phases were tested with the goal of achieving baseline separation within a shorter analysis time. Experimental results indicated that with a mixture of acetonitrile and water $(70+30, v+v)$ as a mobile phase, 4-NP and 4- $t$-OP were baseline separated within $10 \mathrm{~min}$, and the peak of APEOs appeared at the same place as APs. Thus, a mixture of acetonitrile and water $(70+30, v$ $+v$ ) was adopted as mobile phase throughout.

\subsection{MMLLE parameters}

Some MMLLE related parameters such as organic solvent, sample $\mathrm{pH}$, sample flow rate, salt effect, and enrichment time were studied for a $5 \mu \mathrm{g} / L$ 4-NP and 4- $t$-OP standard mixture.

\subsubsection{Solvent used as acceptor}

Three kinds of solvents including dichloromethane, chloroform, and hexane were tested in this study and the results indicated that the highest and the lowest peak height were obtained when dichloromethane and hexane were used, respectively. Unfortunately, when dichloromethane or chloroform was used as acceptor, water in the sample occasionally penetrated through the PTFE membrane and was trapped in the acceptor. This phenomenon was also observed by Norberg et al. [19] when they extracted cationic surfactants by MMLLE. This probably happens because dichloromethane and chloroform are polar solvents with relatively high solubility in water. Therefore, hexane was used in the following studies.

\subsubsection{Sample pH}

4-NP and 4-t-OP are very weak acids with $\mathrm{p} K_{\mathrm{a}}$ around 9.5 [12], which should be protonized before extraction into organic phase. The influence of sample $\mathrm{pH}$ was investigated by adjusting the sample solution to different $\mathrm{pH}$ with sulfuric acid or sodium hydroxide. As shown in Figure 3, the largest peak height was obtained at $\mathrm{pH}$ 6.0. Therefore, $\mathrm{pH} 6.0$ was adopted in the following studies.

\subsubsection{Sample flow rate}

The effect of sample flow rate on peak area was studied at fixed enrichment time and at fixed sample volume, respectively. The result complies with the common findings in membrane extraction, i.e., the most efficient extraction is obtained at low donor flow rate while the maximum enrichment factor is obtained at high donor flow rate. Since adequate sample was available in the present study, a sample flow rate of $7.0 \mathrm{~mL} / \mathrm{min}$, i.e. the highest flow rate the piston pump can deliver, was adopted in our further investigations. Additional studies demonstrated 


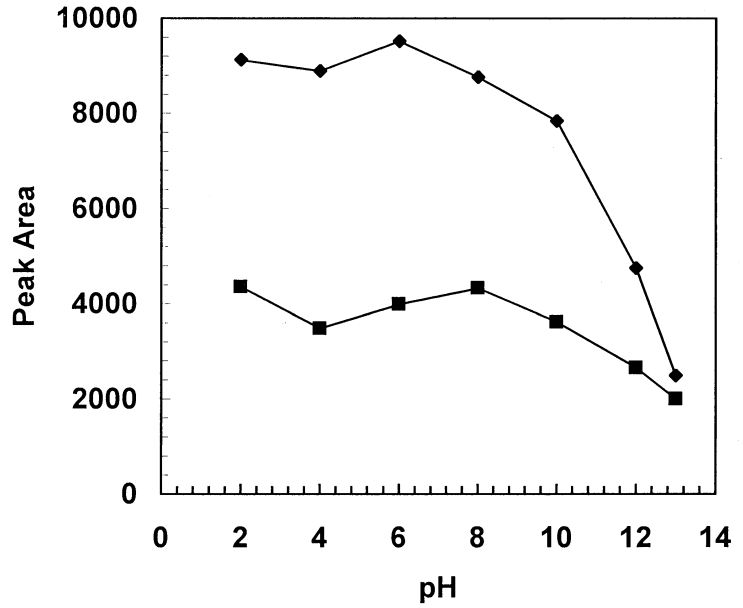

Figure 3. Effect of sample $\mathrm{pH}$ on peak area obtained by enriching $100 \mathrm{~mL}$ of $5 \mu \mathrm{g} / \mathrm{L}$ 4-NP and $4-t-O P$ standard mixture. Sample flow rate, $3.0 \mathrm{~mL} / \mathrm{min}$. See text for experimental conditions. $₫, 4-N P ; ~ 4$, 4-t-OP.

that method precision is independent of the sample flow rate.

\subsubsection{Salt effect}

Addition of salt usually increases the extraction efficiency. To examine the effect of salt addition, different amounts of sodium chloride were added to give $0,5,10,15$, and $20 \%$ $\mathrm{NaCl}$ in the sample solution. The salt concentration did not significantly influence the peak height of 4-NP and 4- $t$-OP. Therefore, no salt was added in the following studies.

\subsubsection{Influence of enrichment time}

With increasing enrichment time, the concentration of analyte trapped in the acceptor increased and gradually approached the equilibrium partition between sample and acceptor, thus affecting the enrichment efficiency. Figure 4 indicates that the peak area of 4-NP and 4-t-OP increased almost linearly with increasing enrichment time in the studied 30-180 min range. This means that there is no saturation of the acceptor and that the equilibrium concentration in the acceptor has still not been reached, even after $180 \mathrm{~min}$ enrichment. This is because the octanol/ water partition coefficients of these analytes are very high $\left(\log K_{\text {ow }}>4\right)$ [20].

\subsubsection{Separation and quantitation of APs and APEOs}

To study the separation ability of the proposed MMLLE procedure, $5 \mu \mathrm{g} / \mathrm{L}$ of $4-\mathrm{NP}$, NP6.5EO, NP10EO, NP15EO, and OP10EO were enriched by the proposed procedure using a sample volume of $180 \mathrm{~mL}$ at a flow rate of $3.0 \mathrm{~mL} /$ min. As shown in Table 1, large amounts of 4-NP and 4-tOP and a small amount of NP6.5EO could be extracted into the acceptor. However, no NP10EO, NP15EO, or

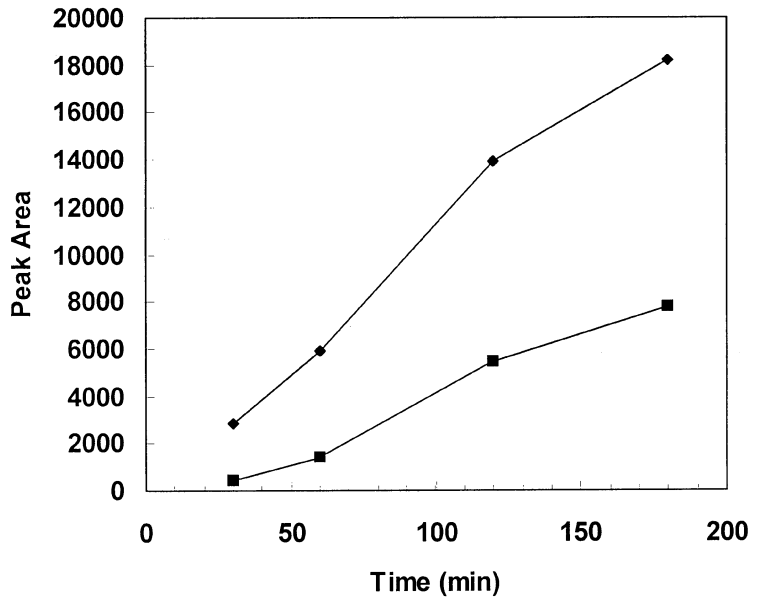

Figure 4. Effect of enrichment time on peak area obtained by enriching $100 \mathrm{~mL}$ of $5 \mu \mathrm{g} / \mathrm{L} \mathrm{4-NP}$ and $4-t$-OP standard mixture. Sample flow rate, $7.0 \mathrm{~mL} / \mathrm{min}$. See text for the other experimental conditions. $\bullet, 4-N P ; \bullet, 4-t-O P$.

Table 1. Peak area of 4-NP, 4-t-OP, and APEOs obtained after MMLLE with a sample volume of $180 \mathrm{~mL}$ at a sample flow rate of $3.0 \mathrm{~mL} / \mathrm{min}$.

\begin{tabular}{lr}
\hline Analyte & Peak Area \\
\hline 4-NP & 9593 \\
NP6.5EO & 350 \\
NP10EO & 10 \\
NP15EO & 0 \\
4-t-OP & 19794 \\
OP10EO & 30 \\
\hline
\end{tabular}

OP10EO was extracted. Clearly, APEOs with EO unit number equal to or larger than 10 can be excluded by the proposed MMLLE procedure. The extraction behavior of SEO-APEOs, with EO unit number of 1-3, were not studied as these standards were not available in our laboratory. According to Table 1, however, it is logical to assume that the extraction recovery of SEC-APEOs is equal to or a little lower than that of APs, and it is impossible to determine APs and SEO-APEOs independently by the proposed MMLLE procedure.

It can be concluded from the above discussion that, when real samples were analyzed, the AP peaks contain both APs and SEO-APEOs, and the quantitative values are minimum values due to the uncertainty of the recovery of SEO-APEOS.

\subsection{Analytical performance}

Under the above selected conditions, some characterisics of the proposed method were investigated. The correlation coefficients $\left(R^{2}\right)$ obtained by determining five 4-NP and $4-t-O P$ standards covering the linear range of $0.1-$ $5 \mu \mathrm{g} / \mathrm{L}$ were 0.9993 and 0.9945 , respectively. The detec- 
Table 2. Amount found in samples and spiked samples (mean $\pm s, n=3$ ) using an enrichment sample volume of $250 \mathrm{~mL}$ by the proposed method.

\begin{tabular}{|c|c|c|c|c|c|c|}
\hline \multirow[t]{2}{*}{ Sample } & \multicolumn{3}{|c|}{ 4-NP } & \multicolumn{3}{|c|}{$4-t-O P$} \\
\hline & Added & Found & $R^{a)}(\%)$ & Added & Found & $R(\%)$ \\
\hline Effluent of sewage & 0 & $0.21 \pm 0.005$ & & 0 & $N D^{b)}$ & \\
\hline treatment works & 0.5 & $0.66 \pm 0.07$ & $89 \pm 14$ & 0.5 & $0.55 \pm 0.05$ & $110 \pm 10$ \\
\hline River water 1 & 0 & ND & & 0 & ND & \\
\hline & 0.5 & $0.45 \pm 0.06$ & $90 \pm 12$ & 0.5 & $0.38 \pm 0.03$ & $76 \pm 6$ \\
\hline River water 2 & 0 & ND & & 0 & ND & \\
\hline & 0.5 & $0.49 \pm 0.08$ & $98 \pm 16$ & 0.5 & $0.37 \pm 0.03$ & $74 \pm 6$ \\
\hline Tap water & 0 & ND & & 0 & ND & \\
\hline & 0.5 & $0.54 \pm 0.08$ & $100 \pm 15$ & 0.5 & $0.42 \pm 0.04$ & $84 \pm 8$ \\
\hline
\end{tabular}

a) Recovery.

b) Not detected.

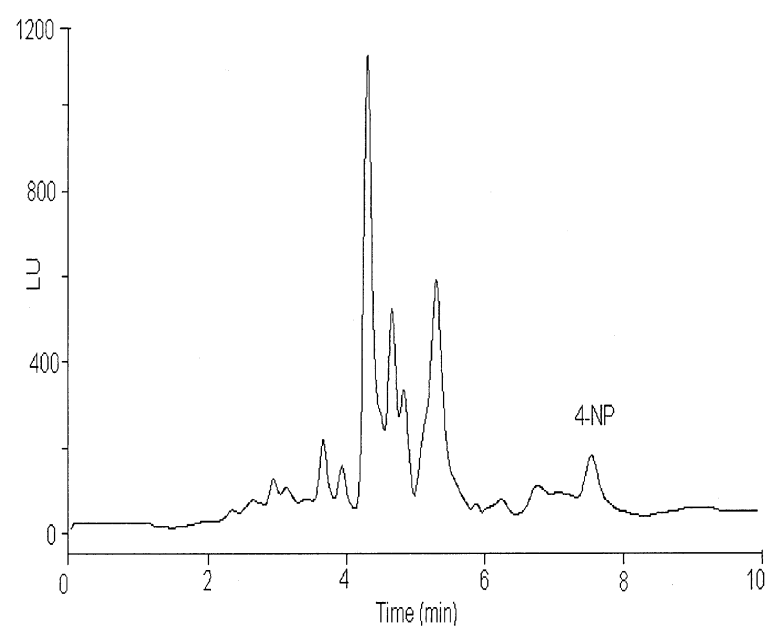

Figure 5. Typical chromatograms of effluent of sewage treatment works.

tion limits $(S / N=3)$ were $0.1 \mu \mathrm{g} / \mathrm{L}$ for $4-N P$ and $0.05 \mu \mathrm{g} / \mathrm{L}$ for 4-t-OP, respectively. The precisions (RSD, $n=5$ ) of the system, measured by repeated enrichment of a standard solution containing $0.5 \mu \mathrm{g} / \mathrm{L}$ 4-NP and 4-t-OP, were $13 \%$ and $10 \%$, respectively.

\subsection{Application of the method to aqueous samples}

In order to validate the proposed method, four aqueous samples (tap water, river water, and effluent from a sewage treatment works) were analyzed and the recoveries were determined at $0.5 \mu \mathrm{g} / \mathrm{L}$ 4-NP and 4-t-OP spiking levels. Results listed in Table 2 indicate that, except in the case of the sewage treatment effluent, which contains $0.21 \mu \mathrm{g} / \mathrm{L}$ 4-NP and SEC-NPEOs as shown in Figure 5, the contents of 4-NP, 4-t-OP, and SEC-APEOs in the other samples were all below the detection limit. The recoveries of 4-NP and 4-t-OP were in the range of $89-$ $108 \%$ and $74-110 \%$, respectively. It must be admitted, however, that the standard deviations were rather high in most instances at $0.5 \mu \mathrm{g} / \mathrm{L}$ spiked level.

\section{Conclusions}

A novel method, based on coupling of MMLLE sample pretreatment and reversed-phase HPLC determination, was proposed for the determination of 4-NP, 4-t-OP, and SEO-APEOs in environmental aqueous samples. After MMLLE, LEC-APEOs were eliminated, while 4-NP, 4-tOP, and SEC-APEOs were enriched and then conveniently determined by reversed-phase HPLC-FLD. The proposed procedure has the advantage of low organic solvent consumption and semi-automation. What is more important, 4-NP or 4-t-OP and SEC-APEOs with short ethoxyl chain, which have similar endocrine activity and appeared as one peak in HPLC, could be readily determined.

\section{Acknowledgements}

This work was jointly supported by the National Natural Science Foundation of China $(20177026,20137010)$ and the Chinese Academy of Sciences (KZCXZ-414).

\section{References}

[1] M. Ahel, W. Giger, M. Koch, Water Res. 1994, 28, 11311142.

[2] T.A. Aboul-Kassim, B.R.T. Simoneit, Crit. Rev. Environ. Sci. Technol. 1993, 23, 325-376.

[3] S. Jobling, J.P. Sumpter, Aquat. Toxicol. 1993, 27, 361372.

[4] S. Jobling, D. Sheahan, J.A. Osborne, P. Matthiessen, J.P. Sumpter, Environ. Toxicol. Chem. 1996, 15, 194-202.

[5] D.Y. Shang, R.W. Macdonald, M.G. Ikonomou, Environ. Sci. Technol. 1999, 33, 1366-1372.

[6] A.T. Kiewiet, P. de Voogt, J. Chromatogr. A 1996, 733, 185-192.

[7] H. Lee, Water Qual. Res. J. Canada 1999, 34, 3-35. 
[8] P. de Voogt, K. de Beer, F. van der Wielen, Trends Anal. Chem. 1997, 16, 584-595.

[9] S.A. Snyder, T.L. Keith, D.A. Verbrugge, E.M. Snyder, T.S. Gross, K. Kannan, J.P. Giesy, Environ. Sci. Technol. 1999, 33, 2814.

[10] T. Takasu, A. Ales, K. Hasebe, Anal. Bioanal. Chem. 2002, 372, 554-561.

[11] P.L. Ferguson, C.R. Iden, B.J. Brownawell, Anal. Chem. 2000, 72, 4322-4330.

[12] A. Motoyama, A. Suzuki, O. Shirota, R. Namba, Rapid Commun. Mass Spectrom. 1999, 13, 2204-2208.

[13] H. Lee, T.E. Peart, Anal. Chem. 1995, 67, 1976.

[14] N.C. van de Merbel, J. Chromatogr. A 1999, 856, 55.
[15] J.Å. Jönsson, L. Mathiasson, J. Chromatogr. A 2000, 902, 205-225.

[16] A.M. Kvistad, E. Lundanes, T. Greibrokk, Chromatographia $1998,48,707$.

[17] M. Ahel, W. Giger, Anal. Chem. 1985, 57, 1577.

[18] K. Inoue, Y. Yoshimura, T. Makino, H. Nakazawa, Analyst 2000, 125, 1959.

[19] J. Norberg, E. Thordarson, L. Mathiasson, J.Å. Jönsson, J. Chromatogr. A 2000, 869, 523-529.

[20] D.T. Bennie, C.A. Sullivan, H.-B. Lee, T.E. Peart, R.J. Maguire, Sci. Total Environ. 1997, 193, 263-275. 